\title{
The Influence of a Combined Strain-Heat Treatment on the Features of Electromagnetic Testing of Fatigue Degradation of Quenched Constructional Steel
}

\author{
A. V. Makarov ${ }^{a}$, E. S. Gorkunov ${ }^{a}$, R. A. Savrai ${ }^{a}$, L. Kh. Kogan ${ }^{b}$, A. S. Yurovskikh ${ }^{c}$, \\ Yu. M. Kolobylin ${ }^{a}$, I. Yu. Malygina ${ }^{a}$, and N. A. Davydova ${ }^{a}$ \\ ${ }^{a}$ Institute of Engineering Science, Ural Branch, Russian Academy of Sciences, \\ Komsomol'skaya ul. 34, Yekaterinburg, 620049 Russia \\ e-mail:makarov@imach.uran.ru,ges@imach.uran.ru,ras@imach.uran.ru,labkm@imach.uran.ru,davydova@imach.uran.ru \\ ${ }^{b}$ Institute of Metal Physics, Ural Branch, Russian Academy of Sciences, \\ ul. Sof'i Kovalevskoi 18, Yekaterinburg, 620041 Russia \\ e-mail:nichip@imp.uran.ru \\ ${ }^{c}$ Ural Federal University, ul. Mira 19, Yekaterinburg, 620002 Russia \\ e-mail: artem.yurovskikh@mail.ru \\ Received July 24, 2013
}

\begin{abstract}
The possibilities of the magnetic and eddy-current methods for testing fatigue degradation during low-cycle loading of quenched steel $50(0.51 \% \mathrm{C})$ that was subjected to a combined strain-heat treatment according to an optimal regime that included friction treatment with subsequent tempering at $T=350^{\circ} \mathrm{C}$, were investigated. It is shown that for steel that was subjected to a combined nanostructuring treatment, the accumulation of a plastic strain under "hard" cyclic loading can be tested using the coercimetric method and values of the residual magnetic induction on the major and minor magnetichysteresis loops, values of the maximum and initial magnetic permeabilities, and readings of an eddy-current instrument at a low excitation frequency of the eddy-current transducer. The appearance of surface fatigue cracks can be tested via eddy-current measurements at high frequencies, when the contribution of the crack formation in the hardened layer to the eddy-current characteristics is considerable.
\end{abstract}

Keywords: constructional steel, strain-heat nanostructuring treatment, low-cycle fatigue loading, magnetic inspection, eddy-current testing

DOI: $10.1134 / \mathrm{S} 1061830913120048$

\section{INTRODUCTION}

The provision of the required serviceability and longevity of articles under specified operational conditions is one of the most important problems of modern machine building. To solve this problem, various surface-treatment techniques are used. This treatment plays a decisive role in providing the working capacity of articles in many cases, e.g., during their operation under cyclic loading. The favorable influence of surface treatments on the operational stability of machine parts may be associated not only with surface-layer hardening but also with the formation of favorable residual compressing stresses in it, as well as with the improvement of the surface treatment finish (roughness reduction).

However, surface hardening may be accompanied by undesirable effects, such as a decrease in plasticity and an increase in the susceptibility to destruction. The use of combined treatment methods that involve the action of several factors on a treated surface is an effective method to eliminate the flaws that are inherent in various methods for surface hardening of parts. The combined strain-heat treatment (SHT), which combines surface strengthening of quenched steel via intense plastic straining using the method of nanostructuring friction treatment and subsequent thermal action, which improves the plastic properties of an ultimately strengthened material, can be classified as such treatments [1, 2].

In several previous studies, it was established that eddy-current (EC) and magnetic characteristics can be used as testing parameters of quenched medium- and high-carbon steels that are subjected to frictional treatment and combined SHTs, which include frictional treatment and tempering at temperatures of 100$600^{\circ} \mathrm{C}[3-5]$. In particular, it was shown that using the coercive force, residual magnetic induction, the maximum magnetic permeability, and readings of an EC instrument, unambiguous testing of the struc- 
tural state of quenched constructional steel 50, which was subjected to an optimal SHT, is possible. This SHT includes frictional treatment with tempering at $350^{\circ} \mathrm{C}$ and, in comparison to thermal treatment (quenching with tempering at the same temperature), it provides an increase in the hardness and wear resistance under frictional and abrasive actions by a factor of $2-2.5$ in the absence of a decrease in mechanical properties (including plasticity) under static tension [5].

It was proposed to use the magnetic, EC, and electromagnetic-acoustic methods for evaluating the accumulated strain and forecasting fracturing under cyclic loading of carbon steels with ferrite-pearlite structures [6-9], thermostrengthened chromium steel [10], and annealed low-carbon steel that was strengthened by frictional treatment [11].

The objective of this study was to investigate the possibilities of magnetic and EC methods when testing the fatigue degradation under low-cycle fatigue loading of quenched steel $50(0.51 \% \mathrm{C})$. This steel was subjected to combined SHT according to an optimal regime, which includes a frictional treatment with a sliding indenter with subsequent tempering at $T=350^{\circ} \mathrm{C}$, providing a good combination of the wear resistance, strength, and plasticity of the constructional steel.

\section{THE MATERIAL AND EXPERIMENTAL TECHNIQUE}

Specimens of steel $50(0.51 \% \mathrm{C})$ were quenched from $850^{\circ} \mathrm{C}$ in $\mathrm{I}-12$ industrial oil (quenching in oil prevented the appearance of quenching cracks). After the quenching, a combined SHT was performed, which included frictional treatment with subsequent tempering in a vacuum at $T=350^{\circ} \mathrm{C}$ (holding for $1 \mathrm{~h})$. The frictional treatment of the working part $(25 \times 7 \mathrm{~mm})$ of $2.7-\mathrm{mm}$-thick flat specimens was performed in air during scanning (with a transverse displacement) with a hemispherical VK-8 hard-alloy indenter with a radius of the hemisphere of $2.5 \mathrm{~mm}$ at a load of $588 \mathrm{~N}$ and an average sliding velocity of $0.026 \mathrm{~m} / \mathrm{s}$. The form of the specimens and scheme of their frictional treatment are presented in [4]. Two sides of the flat specimens were subjected to the frictional treatment for subsequent mechanical tests and physical measurements. In order to compare the thermal and SHT treatments, some specimens were quenched and then tempered at $T=350^{\circ} \mathrm{C}$ for $1 \mathrm{~h}$.

Mechanical tests for static and cyclic tensions were performed on an Instron 8801 facility. The cyclic loading was conducted at a controlled value of the total strain $\varepsilon_{\mathrm{tot}}=2 \varepsilon_{\mathrm{a}}=\varepsilon_{\mathrm{el}}+\varepsilon_{\mathrm{pl}}=0.022\left(\varepsilon_{\mathrm{a}}\right.$ is the amplitude of the total strain in a cycle, $\varepsilon_{\mathrm{el}}$ is the amplitude of the elastic strain in a cycle, and $\varepsilon_{\mathrm{pl}}$ is the amplitude of the plastic strain in a cycle), a cycle-asymmetry coefficient $R_{\varepsilon}=0$ (constant-sign tension from zero), a change in the strain amplitude in the cycle according to a sawtooth law, and a loading frequency of $0.5 \mathrm{~Hz}$. In this case, tests were accomplished so that the cycle strain $\left(R_{\varepsilon}\right)$ and stress $\left(R_{\sigma}\right)$ asymmetry coefficients remained equal to zero during the entire loading process $\left(R_{\sigma}=R_{\varepsilon}=0\right)$. Specimens were tested with numbers of loading cycles $N=10,50,200,900$, and 1200 . The value of the accumulated plastic strain was calculated using the formula $\varepsilon_{\Sigma}=\ln \left(l_{k} / l_{0}\right)$, where $l_{0}$ is the calculated specimen length and $l_{k}$ is the gage length of a specimen after the cyclic loading.

The surfaces of specimens were studied by the scanning electron microscopy (SEM) method using a Tescan VEGA II XMU microscope. The parameters of the surface roughness of specimens were determined using a Wyko NT-1100 optical profilometer on five areas with dimensions of $42.5 \times 55.8 \mu \mathrm{m}$. The fine structure was investigated using the transmission electron microscopy (TEM) method on a JEOL JEM-2100 microscope with application of one- and two-sided mechanical and electrolytic thinning of blanks (foils). The microhardness was measured on a Leica VMHT microhardness tester with an indenter load of $0.245 \mathrm{~N}$. The width of the (110) $\alpha \mathrm{X}$-ray line was determined using a SHIMADZU XRD-7000 $\mathrm{X}$-ray diffractometer in $\mathrm{Cr}-K_{\alpha}$ radiation.

The magnetic characteristics were measured on a Remagraph C-500 magnetic-measurement system on the major and minor magnetic-hysteresis loops. The coercive force and residual induction were recorded for the major loop $\left(H_{\max }=60 \mathrm{kA} / \mathrm{m}\right)$ and minor hysteresis loops corresponding to the maximum magnetic inductions in hysteresis cycles $b_{\max }=1,0.4,0.1$, and $0.05 \mathrm{~T}$; the maximum magnetic permeability $\mu_{\max }$ was also measured. The value of the initial permeability $\mu_{\text {in }}$ was determined by calculations. The dependence $\mu(H)=B / \mu_{0} H$, where $B$ is the magnetic induction, T, and $H$ is magnetic-field strength, $\mathrm{A} / \mathrm{m}$, was plotted for the initial segment of the magnetization curve [12]. The value of $\mu_{\text {in }}$ was determined via a linear extrapolation of the dependence $\mu(H)$ to $H=0$.

The value of the resistivity $\rho$ was determined indirectly using a BS3-010-2 microohmmeter. In this case, the electrical resistance $R$ was measured on a segment with the specified length $l=22 \mathrm{~mm}$ within the gage length of the specimens having a constant cross section with the area $S$. After this, the resistivity was calculated from the formula $\rho=R S / l$. 

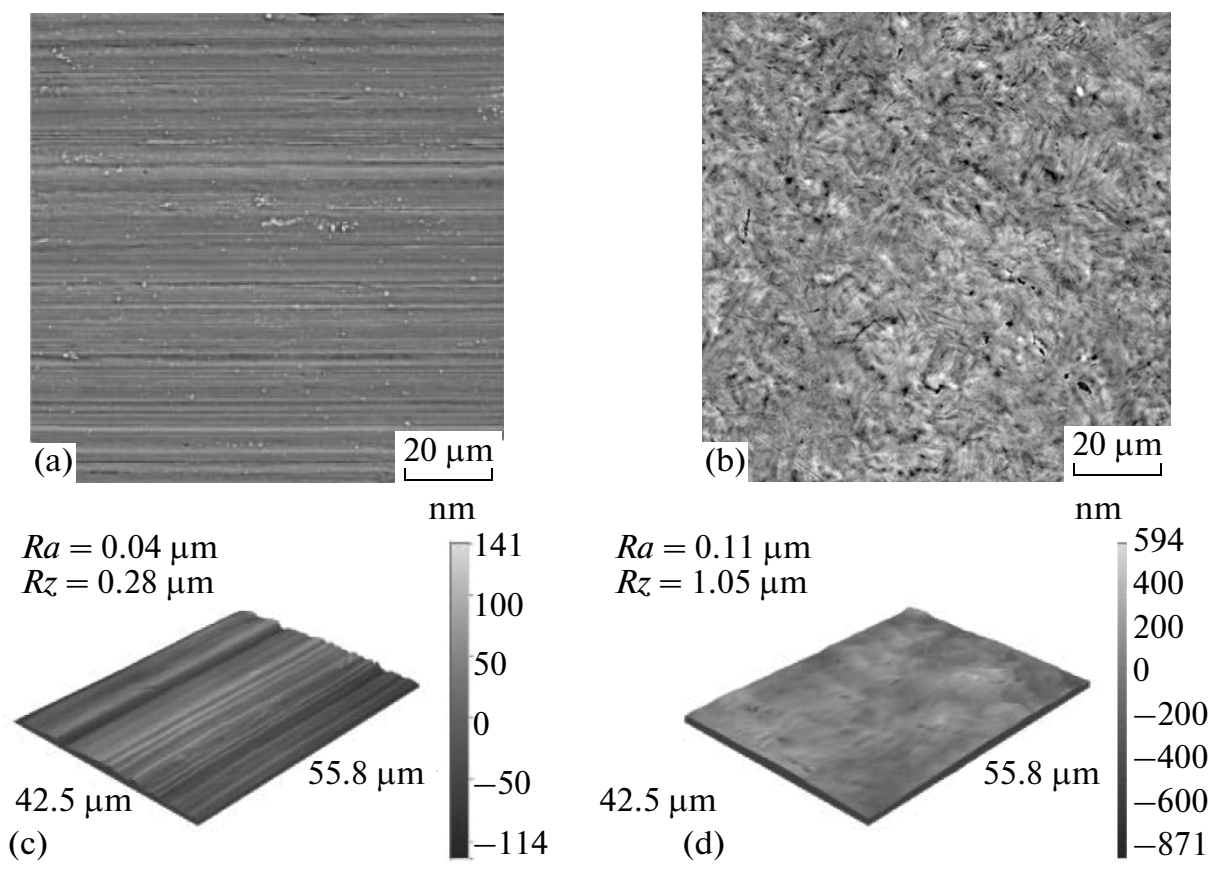

Fig. 1. The characteristics of the surface of steel 50 after (a, c) combined SHT and (b, d) heat treatments: $(a, b)$ images on a scanning electron microscope and (c, d) 3D images on an optical profilometer.

The electromagnetic parameters were measured on a laboratory prototype of an EC instrument using a differentially connected attachable transformer-type transducer with a ferrite cup core at frequencies $f=2.4,12,24,36,72,96$, and $108 \mathrm{kHz}$. A schematic image of the used transducer with a flat side surface (the dimensions of the current winding and cores are given) are presented in [13]. The locality of the transducer made it possible to perform measurements on the lateral surfaces of the working part of a specimen ( $7 \mathrm{~mm}$ wide) without an edge effect (the testing locality was 5-6 $\mathrm{mm}$ in diameter). Each reading of the EC instrument was found as the arithmetic mean of six measurements.

\section{EXPERIMENTAL RESULTS AND DISCUSSION}

Influence of the combined strain-heat treatment on the surface roughness, structure, and microhardness of quenched steel 50. As a result of a combined SHT (frictional treatment + tempering at $350^{\circ} \mathrm{C}$ ) of quenched steel 50 specimens, a smoothed surface with roughness parameters $R a=0.04 \mu \mathrm{m}$ and $R z=0.28 \mu \mathrm{m}$ (Fig. 1a, 1c) was formed. On the considered friction surface, traces of the material, which was pushed aside, in the form of small longitudinal recesses and protrusions were observed. Note that no microcracks of the fatigue nature were observed on the surface of steel 50 (low-cycle friction fatigue) [14]. Such microcracks were observed after the frictional treatment of Ст3 steel with a hard-alloy cylindrical indenter [15]. This is due to the choice of the frictional-treatment parameters, for which efficient strengthening of the surface layer is provided, and the degree of metal damage, which is initiated by a large plastic strain [16], does not reach the critical values that result in a destruction of material. Light-colored particles that also occur on the surface (Fig. 1a) represent the indenter material (BK-8 alloy that consists of tungsten carbides and a cobalt binder), which was brought to the steel surface during frictional loading [15].

The surface of steel 50 that was subjected only to a heat treatment (quenching + tempering at $350^{\circ} \mathrm{C}$ ) has a smoothed relief with etching pits (Fig. 1b, 1d), which are typical for an electropolished surface, and is characterized by a much higher roughness: $R a=0.11 \mu \mathrm{m}$ and $R z=1.05 \mu \mathrm{m}$.

Electron-microscopic investigations showed that a relatively homogeneous disperse structure (Fig. 2a) with an average crystal size in the $\alpha$-phase that is usually within $100 \mathrm{~nm}$ (Fig. 2b) appears in a thin surface layer of quenched steel 50 as a result of the combined SHT. The presented microelectronogram (Fig. 2a) in the form of an almost continuous ring, which is formed by reflections from individual crystallites that are close to one another, provide evidence of their large-angle disorienrations. Hence, the considered structure can be classified as a nanocrystalline structure and the combined SHT that forms the above structure is a nanostructuring treatment procedure. 

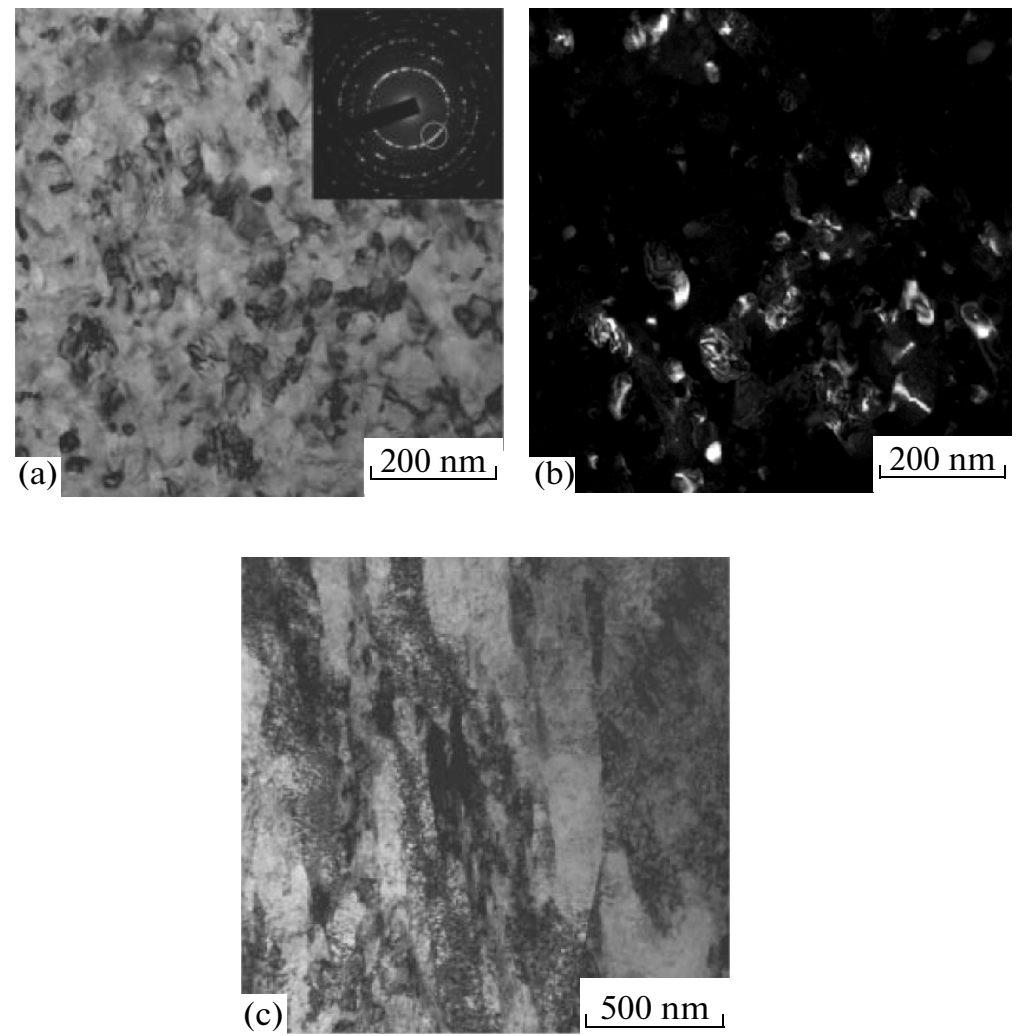

Fig. 2. The structure (TEM) of steel 50 after the (a, c) combined SHT (frictional treatment + tempering at $350^{\circ} \mathrm{C}$ ) and (c) heat treatment (quenching + tempering at $\left.350^{\circ} \mathrm{C}\right)$ : $(\mathrm{a}, \mathrm{c})$ light-field images and (b) a dark-field image in the (110) $\alpha$ reflection.

A nanocrystalline structure arises in a thin $(5-8 \mu \mathrm{m})$ surface layer of a quenched steel that is subjected to frictional treatment, which leads to fragmentation of the initial structure of packet and laminated martensite and rotations of fragments [17]. Tempering at $350^{\circ} \mathrm{C}$ (for $1 \mathrm{~h}$ ) causes a definite increase in the dimensions of $\alpha$ phase crystallites, but the structure's dispersivity holds at a rather high level (Fig. 2a, 2b). A decrease in the dislocation density inside crystallites and a decrease in the azimuthal broadening of $\alpha$-phase reflections on the microelectronogram, which are observed after the tempering, indicate that reverse processes occur. This is displayed as an almost twofold decrease in the integral width of the (110) $\alpha$ X-ray line for tempered steel, which was subjected to the frictional treatment (see the table). However, the aforementioned structural modifications determine only a relatively small decrease (from 1060 to $1000 \mathrm{HV} 0.025$ ) in the microhardness of the steel that was strengthened with the frictional treatment after tempering at $350^{\circ} \mathrm{C}$ (see the table). It was shown in [4] that when quenched steel is friction treated, the maximum levels of the microhardness and width of the $\mathrm{X}$-ray line are observed directly on the material surface, where the highest degree of accumulated plastic strain is attained $[15,16]$. The maximum decrease in the microhardness of steel that was subjected to frictional treatment occurs in a $\sim 30-\mu \mathrm{m}$-thick layer and the total thickness of the frictional-treatment-hardened layer for a specimen that was quenched in oil is $\sim 220 \mu \mathrm{m}$ [4].

Figure $2 \mathrm{c}$ shows that after a standard heat treatment (quenching + tempering at $350^{\circ} \mathrm{C}$ ), a typical macrocrystalline structure of tempered martensite arises in steel 50. Martensite preserves the morphological features of the initial quenched structure, which consists of laminated and packet martensites [4, 17].

Microhardness $H V 0.025$ and integral width $B$ of the (110) $\alpha$ X-ray line for steel 50 after various treatments

\begin{tabular}{l|r|r}
\hline \multicolumn{1}{c|}{ Treatment } & $H V 0.025$ & $B, \min$ \\
\hline Quenching from $850^{\circ} \mathrm{C}$ in oil + frictional treatment & $1060 \pm 35$ & 72.0 \\
Combined SHT of quenched steel (frictional treatment + tempering at $350^{\circ} \mathrm{C}$ ) & $1000 \pm 30$ & 37.5 \\
Heat treatment (quenching + tempering at $350^{\circ} \mathrm{C}$ ) & $460 \pm 10$ & 30.5 \\
\hline
\end{tabular}


(a)

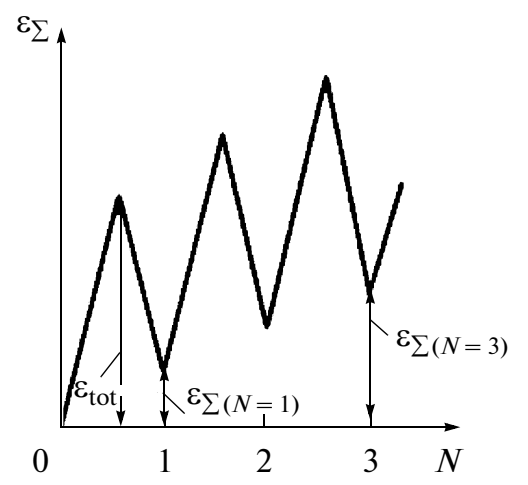

(b)

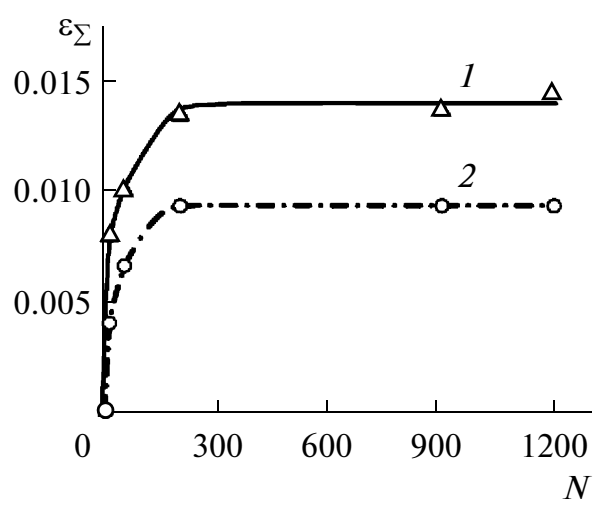

Fig. 3. (a) Schemes of cyclic loading with a controlled value of the total strain $\varepsilon_{\text {tot }}$ and (b) the dependence of the accumulated plastic strain $\varepsilon_{\Sigma}$ on the number of loading cycles $N$ under cyclic loading of steel 50: (1) after heat treatment (quenching + tempering at $350^{\circ} \mathrm{C}$ ) and (2) after combined SHT of quenched steel (frictional treatment + tempering at $350^{\circ} \mathrm{C}$ ).

Cementite in the form of extended (lath-shaped) and rounded precipitations deposits along the boundaries of and inside martensite crystals as a result of $1-\mathrm{h}$ tempering at $T=350^{\circ} \mathrm{C}$. The microhardness of steel after quenching and tempering at $T=350^{\circ} \mathrm{C}$ is $460 H V 0.025$; this value is lower than the hardness of steel after the combined SHT by a factor of $>2$ (see the table). After the considered heat treatment, steel is also characterized by the smallest width of the (110) $\alpha \mathrm{X}$-ray line $(B=30.5 \mathrm{~min}$, see the table $)$ and, correspondingly, the lowest levels of defects in the crystal structure and microdistortions in the $\alpha$ phase.

Influence of the combined SHT on the features of strain and destruction of quenched steel 50 in a cyclic loading process. Figure 3 shows that under the used conditions of cyclic loading with a controlled value of the total strain $\varepsilon_{\text {tot }}=0.022$ (Fig. 3a), the accumulation of a plastic strain in specimens that were subjected to heat (Fig. 3b, curve 1) and SHT treatments (Fig. 3b, curve 2) occurs only in the first 200 tension cycles (from zero). When the number of cycles increases to 900 and 1200, no additional increase in the value of the accumulated plastic strain is observed as a result of strain hardening of steel and further straining occurs only in the elastic region. It also follows from Fig. $3 \mathrm{~b}$ that after the SHT, a smaller maximum plastic strain $\left(\varepsilon_{\Sigma}=0.0093\right.$, curve 2$)$ is accumulated in steel under cyclic loading than after heat treatment $\left(\varepsilon_{\Sigma}=\right.$ 0.0145 , curve 1 ). This is determined by the presence of a highly strained layer, which was hardened during the frictional action and is less capable of being plastically deformed, on the steel surface.

Figure 4a shows that during "hard" fatigue loading (with a constant-amplitude strain being maintained in each cycle), the microhardness of steel 50 after heat treatment and the combined SHT remains virtually constant during the entire loading process. However, the width of the X-ray line of the $\alpha$ phase of steel 50 appreciably changes (Fig. 4b). At the initial loading stage (up to ten loading cycles for specimens after the standard heat treatment and up to 50 loading cycles for specimens after the combined SHT), an abrupt increase in the linewidth is observed, which reflects an increase in the density of structural defects. Then, after up to 200 loading cycles are performed, a decrease in the widths of the X-ray lines is observed, which may be associated with a saturation of the dislocation density and redistribution of dislocations inside dislocation substructures that formed during cyclic straining $[18,19]$, as well as with an outflow of defects to submicroscopic continuity flaws. As the cyclic loading continues, the linewidths remain virtually constant at levels of $\sim 33 \mathrm{~min}$ (specimens after heat treatment) and $\sim 40 \mathrm{~min}$ (specimens after combined SHT), which exceed the linewidths before the cyclic loading (Fig. 4b). Note that before the cyclic loading, the linewidth of the $\alpha$ phase of steel 50 for specimens that were subjected to the SHT was 7 min larger than that for specimens that were subjected to the standard heat treatment. This difference remained constant even after the cyclic loading.

Studying the surfaces of specimens after cyclic tension shows (Fig. 5) that the combined SHT substantially modified the character of the development of the material plastic flow during straining. Cracks, which are caused by the action of normal stresses and whose length and number increase with an increase in the number of loading cycles, are observed on the surfaces of specimens that were subjected to the SHT (Fig. 5a, 5b). The appearance of cracks on the steel surface with a gradient surface layer is caused by abrupt differences in the properties of the metal on the specimen surface and in the bulk. When such specimens are strained, quasi-periodic stress mesoconcentrators that appear at the surface-hardened layer-substrate 

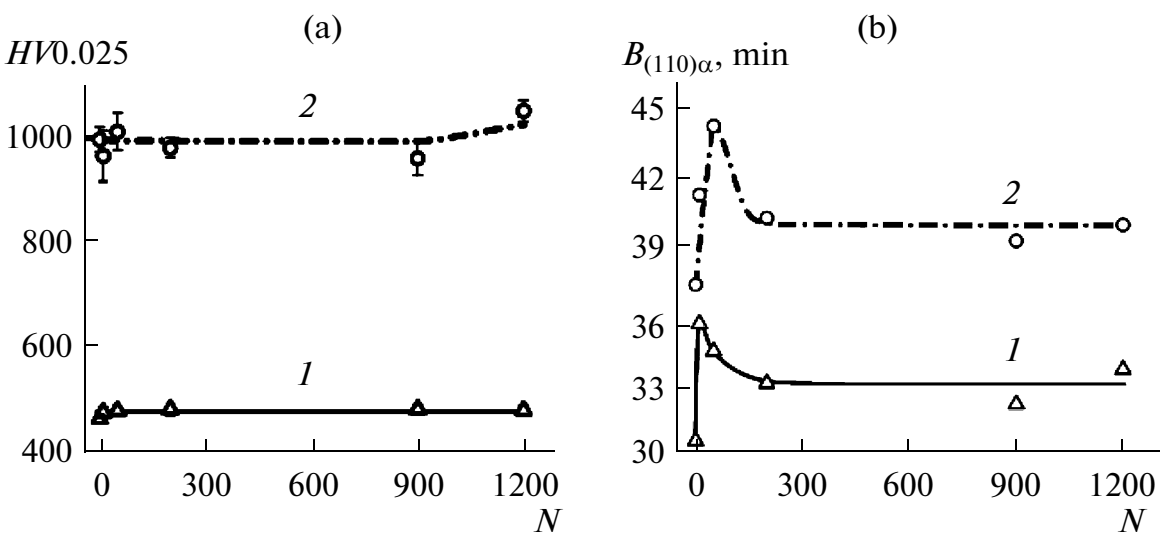

Fig. 4. The influence of cyclic loading on (a) the microhardness, $H V 0.025$, and (b) width $B$ of the (110) $\alpha$ X-ray line of steel 50: (1) after heat treatment (quenching + tempering at $350^{\circ} \mathrm{C}$ ) and (2) after combined SHT of quenched steel (frictional treatment + tempering at $\left.350^{\circ} \mathrm{C}\right)$.

interface are added to the primary stress mesoconcentrators. The relaxation of the peak values of these stresses in the hardened surface layer occurs exactly via the formation of a quasi-periodic system of surface microcracks [20] that appear already after ten loading cycles (Fig. 5a).

On the surfaces of the steel 50 specimens that were subjected to heat treatment, a strain relief (Fig. 5c), which is caused by the effects of shear and rotational strain modes (mesovortices are formed) in the material bulk, is observed at the initial straining stage (for $N=10$ ) [21]. Cracking, which is characteristic of specimens that were subjected to the combined SHT, is not observed. When the number of loading cycles is $N=200$ or more, separate fatigue cracks (Fig. 5d) appear on the surface, which evidently result from zigzag shears, viz., localized-strain mesobands, which propagate along the conjugate directions of the maximum tangential stresses [22]. As a result of relaxation of stresses that are produced during the formation of mesovortices, pores also appear (shown with arrows in Figs. 5c, 5d) on the surface of heat-treated steel after cyclic loading [22].

The influence of the combined SHT on the features of changes in the electromagnetic characteristics of quenched steel 50 under cyclic loading. Figure 6 shows that the dependences of the coercive force on the number of loading cycles (values of the accumulated plastic strain) for steel 50 specimens after the heat and combined SHT treatments are qualitatively similar. The character of these curves depends on the field value. During the magnetization reversal in high $\left(b_{\max } \geq 1 \mathrm{~T}\right)$ and medium $\left(b_{\max }=0.4 \mathrm{~T}\right)$ magnetic fields at the initial stage of fatigue loading for the number of cycles $N=10$ (the value of the accumulated plastic strain is $\varepsilon_{\Sigma}=0.0079$ for specimens after heat treatment and $\varepsilon_{\Sigma}=0.004$ for specimens after the combined SHT), an intense increase in the coercive force is observed. As the cyclic loading continues, the abrupt increase in the coercive force is replaced by its smooth increase and stabilization at the number of cycles $N=200$ (the value of the accumulated plastic strain is $\varepsilon_{\Sigma}=0.0134$ for specimens after heat treatment and $\varepsilon_{\Sigma}=0.0093$ for specimens after the combined SHT), which holds until the end of the loading procedure $(N=1200$ cycles $)$.

The aforementioned character of changes in the coercive force during magnetization reversal in high $\left(b_{\max } \geq 1 \mathrm{~T}\right)$ and medium $\left(b_{\max }=0.4 \mathrm{~T}\right)$ magnetic fields (Fig. 6) properly correlates with the behavior of the dependences of the accumulated plastic strain $\varepsilon_{\Sigma}$ on the number of loading cycles (Fig. $3 \mathrm{~b}$ ), which characterize the change in the total density of defects in steel during cyclic tension to a higher degree than the width of the (110) $\alpha$ X-ray lines (Fig. 4b). In fact, a redistribution of defects during fatigue loading and their outflow to submicroscopic continuity flaws are not accompanied by a decrease in the total density of defects in steel but determine a decrease in the width of the $\alpha$-phase line, as the number of loading cycles exceeds ten for specimens after heat treatment and 50 for specimens after the combined SHT (Fig. 4b). Thus, a continuous increase in the magnetic hardness in strong and medium fields (Fig. 6), which is observed upon an increase in the number of loading cycles to $N=200$, is determined by an increase in the total density of defects. The latter causes an increase in the gradients of microstresses. This leads to higher values of the critical field of the interaction of the domain boundaries with defects, thus correspondingly impeding the magnetization-reversal processes [23, 24]. It is important that the magnetic method makes it possible to test the strain value and the level of the total density of defects, which were stored during 
(a)

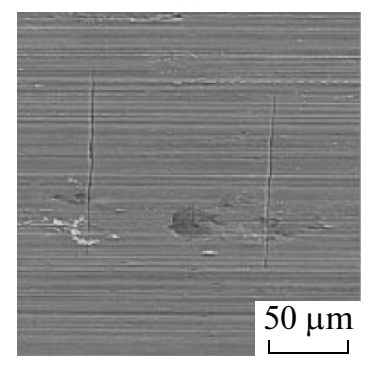

(c)

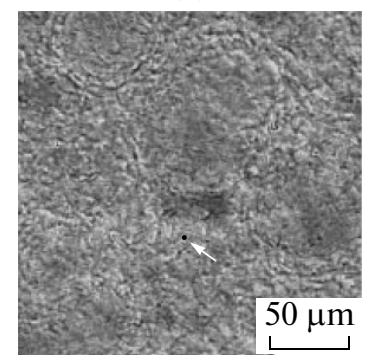

(b)

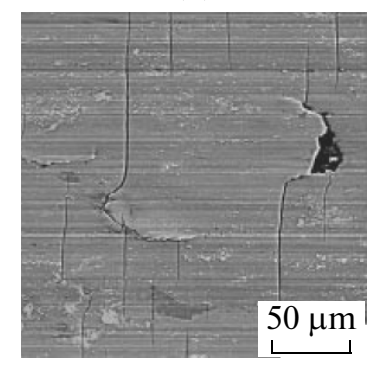

(d)

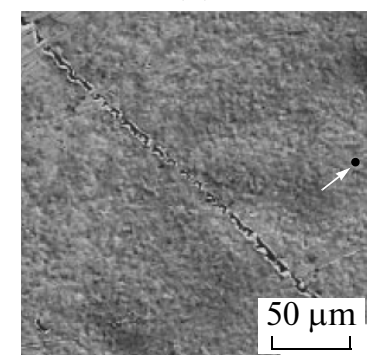

Fig. 5. Electronic microphotos (SEM) of surfaces of samples from steel 50 after combined SHT (a, b) and heat (c, d) treatments subjected to cyclic loading with a number of cycles $N=10(\mathrm{a}, \mathrm{c})$ and $900(\mathrm{~b}, \mathrm{~d})$

cyclic tension (from zero) of steel 50 that was subjected to both the nanostructuring SHT and standard heat treatment, more reliably than the X-ray method.

Figure 6 also shows that as a result of cyclic loading the coercive-force values $H_{c}, h_{c 1}$, and $h_{c 0.4}$ for steel after heat treatment (quenching + tempering at $350^{\circ} \mathrm{C}$ ) increase to higher levels than those for steel after SHT. This follows from the higher value of the accumulated plastic strain for steel after heat treatment (Fig. 3b, curve 1) in comparison to steel after SHT (Fig. 3b, curve 2). The elevated levels of the coercive force for steel after heat treatment in measurements in strong and medium fields are also favored by the appearance of pores (shown with arrows in Fig. 4c, 4d) on its surface after the cyclic loading. Being sources of leakage magnetic fields, the pores prevent magnetization-reversal processes.

In measurements in weak fields (for the maximum magnetic induction in a hysteresis cycle $b_{\max }=0.05$ and $0.1 \mathrm{~T}$ ), the coercive force abruptly decreases at the number of cycles $N=10$ (Fig. 6). Further loading leads to coercive-force stabilization. The decrease in the coercive force of minor cycles, which were measured in weak fields, that was observed at the initial loading stage may be determined by the transformation of a complex $90^{\circ}$ secondary domain structure [25]. This structure arises in ferromagnetic regions with high local stresses and exerts a predominating influence on the magnetization-reversal processes in weak fields [26]. An increase in the defectiveness upon loading promotes the formation of a significant volume of $90^{\circ}$ domain boundaries, for which strong critical fields of interaction between the domain boundaries and defects are characteristic. These fields exceed the critical fields of magnetic structures with $180^{\circ}$ domains by a factor of $\sim 1.5$ [27]. In weak magnetic fields, the $90^{\circ}$ boundaries can only be elastically displaced to small distances, virtually without being detached from their fixation points. Thus, an abrupt decrease in $h_{c 0.05}$, and $h_{c 0.1}$ for the number of loading cycles $N=10$ is determined by a decrease in the probability of irreversible displacements of domain boundaries in weak magnetic fields, thus being a consequence of the reduced role of irreversible magnetization-reversal processes.

As for the case of the coercive force, the dependences of the residual magnetic induction on the number of loading cycles (the value of the accumulated plastic strain) for steel 50 specimens after the heat and combined SHT treatments are qualitatively similar (Fig. 7). However, their character does not depend on the field value. The residual magnetic induction for both the major and minor magnetic-hysteresis loops abruptly decreases at the initial stage of fatigue loading for the number of loading cycles $N=10$ (the value of the accumulated plastic strain is $\varepsilon_{\Sigma}=0.0079$ for the heat-treated specimens and $\varepsilon_{\Sigma}=0.004$ for specimens that were subjected to combined SHT). The further cyclic loading leads to a smooth decrease and stabilization of the residual magnetic induction during the magnetization reversal in high $\left(b_{\max } \geq 1 \mathrm{~T}\right)$ and medium $\left(b_{\max }=0.4 \mathrm{~T}\right)$ fields for the number of cycles $N=200$ (the value of the accumulated plastic strain is $\varepsilon_{\Sigma}=0.0134$ for the heat-treated specimens and $\varepsilon_{\Sigma}=0.0093$ for the specimens subjected to the combined 
(a)

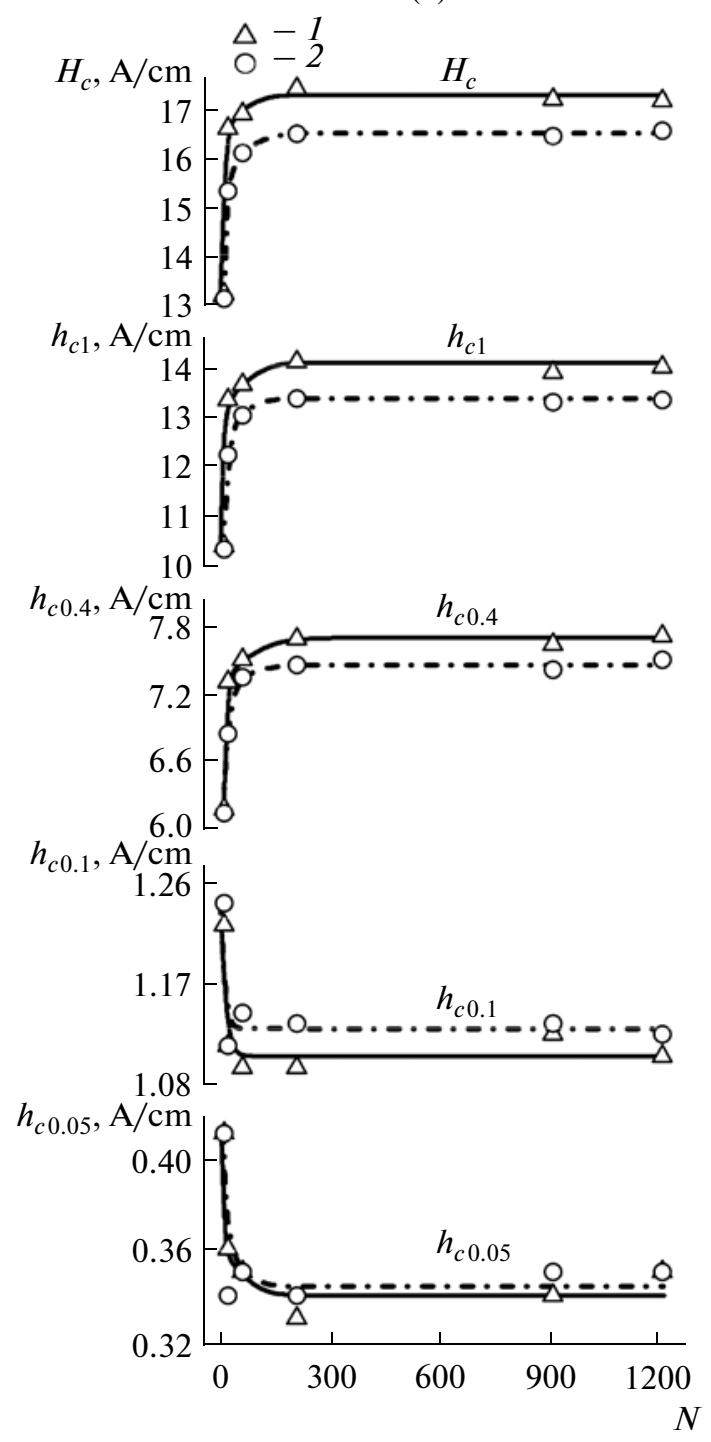

(b)
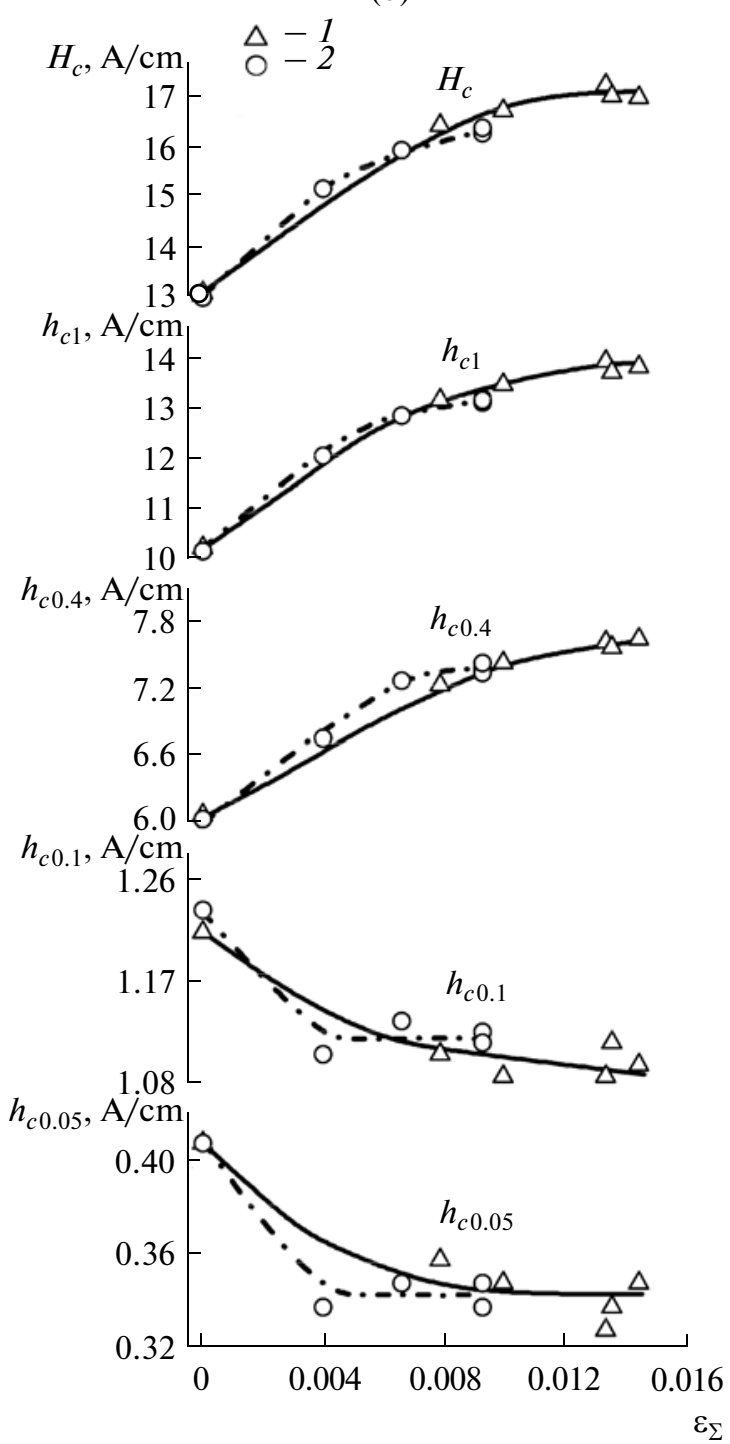

Fig. 6. The dependences of the coercive force on (a) the number of loading cycles and (b) the value of the accumulated plastic strain under cyclic loading of steel 50: (1) after heat treatment (quenching + tempering at $350^{\circ} \mathrm{C}$ ) and (2) after combined SHT of quenched steel (frictional treatment + tempering at $350^{\circ} \mathrm{C}$ ).

SHT). A decrease in the residual induction at the initial stages of cyclic loading (Fig. 7) is determined by an increase in the possible sites of easy nucleation of the reverse magnetic phase as a result of the increasing density of defects in steel. It is known [23] that the process of the formation of residual magnetization in a ferromagnet is largely determined by the number of nucleation centers of reverse magnetic phases.

It follows from Figs. 8 and 9 that the maximum $\mu_{\max }$ and initial $\mu_{\text {in }}$ magnetic permeabilities for steel 50 abruptly decrease at the initial loading stage for the number of cycles $N=10$ (the value of the accumulated plastic strain is $\varepsilon_{\Sigma}=0.0079$ for the heat-treated specimens and $\varepsilon_{\Sigma}=0.004$ for the specimens subjected to the combined SHT). Further cyclic loading leads to a smooth decrease and stabilization of the maximum and initial permeabilities in most cases for the number of cycles $N=50\left(\varepsilon_{\Sigma}=0.01\right.$ for the heat-treated specimens and $\varepsilon_{\Sigma}=0.0066$ for the specimens after combined SHT).

A significant increase in the coercive force and a decrease in both the residual magnetic induction and the maximum and initial permeabilities, observed in Figs. 6-9, may be determined not only by an increase in the density of crystal-structure defects at the initial cyclic-tension stages but also by the appearance of significant residual compressive stresses during unloading of plastically deformed specimens [28, 29]. 
(a)

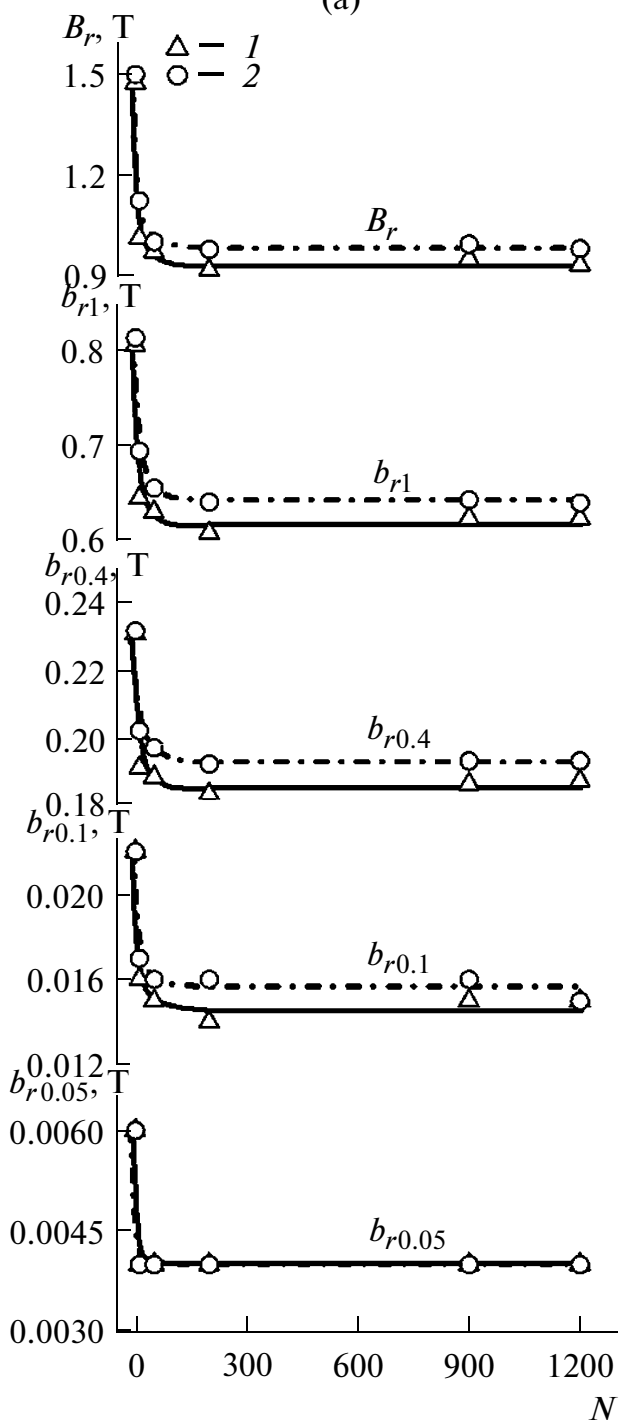

(b)

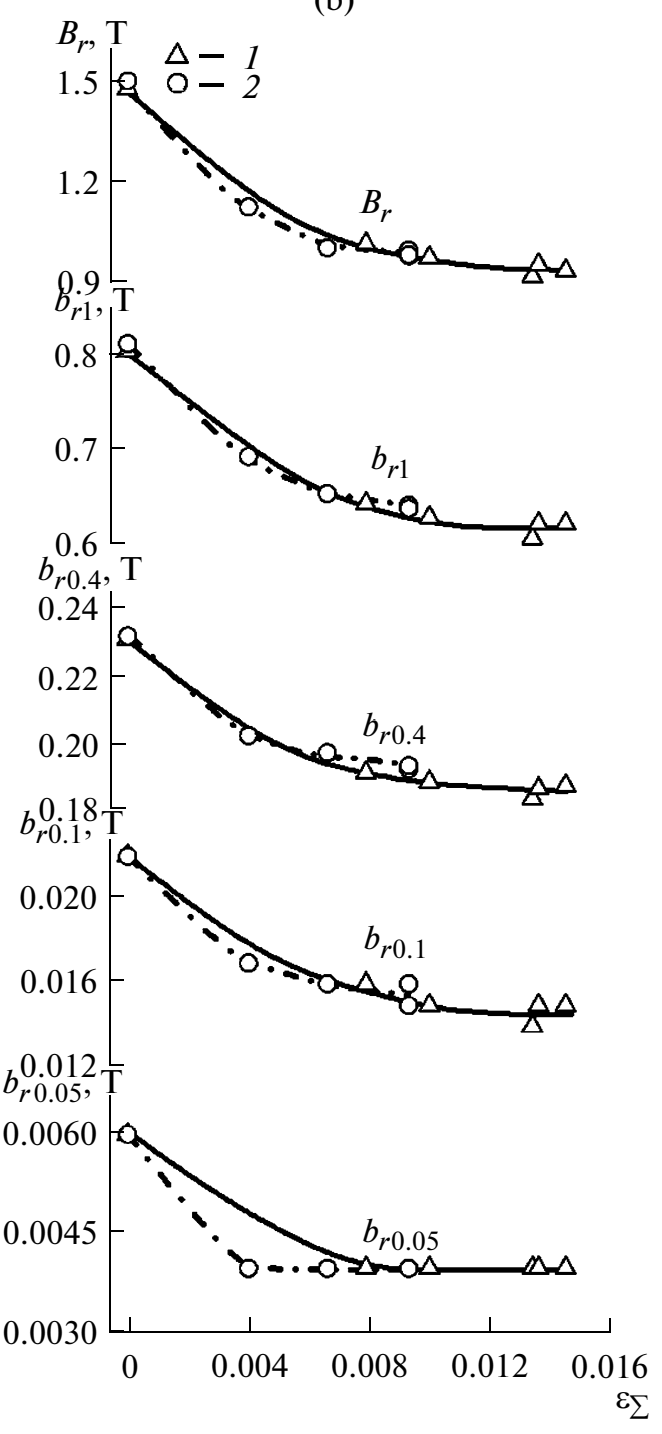

Fig. 7. The dependences of the residual magnetic induction force on (a) the number of loading cycles and (b) the value of the accumulated plastic strain under cyclic loading of steel 50: (1) after heat treatment (quenching + tempering at $350^{\circ} \mathrm{C}$ ) and (2) after combined SHT of quenched steel (frictional treatment + tempering at $350^{\circ} \mathrm{C}$ ).

In contrast to the magnetic characteristics, the resistivity of steel 50 that was subjected to heat and combined SHT treatments underwent insignificant changes under cyclic loading (Fig. 10).

It is important that the magnetic characteristics and electrical resistivity were determined for the entire volume of the working part of 2.7-mm-thick flat specimens, for which the fraction of the efficient and general hardening was 2.2 and $16 \%$ after the frictional treatment of two sides with an effective hardening depth of $\sim 30 \mu \mathrm{m}$ and a total hardening depth of $\sim 220 \mu \mathrm{m}$, respectively. This may also explain the relatively slight differences in the character of changes in the absolute values of the magnetic characteristics and resistivity under cyclic loading of specimens after the combined SHT (including the frictional treatment) and standard heat treatment (Figs. 6-10).

Figure 11 demonstrates the influence of cyclic loading of steel 50 on the readings $\alpha$ of the EC instrument. It follows from Fig. 11 that the dependences of readings $\alpha$ of the EC instrument on the number of loading cycles (the value of the accumulated plastic strain) for steel 50 specimens after the heat and combined SHT treatment are very different. After heat treatment, these dependences have a qualitatively identical character for all excitation frequencies of the EC transducer (Fig. 11, curves 1): at the initial loading stage with the number of cycles $N=10$ (the value of the accumulated plastic strain is $\varepsilon_{\Sigma}=0.0079$ ), the 


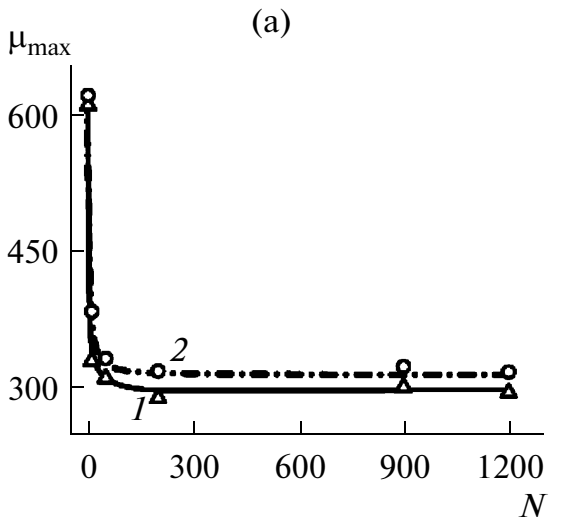

(b)

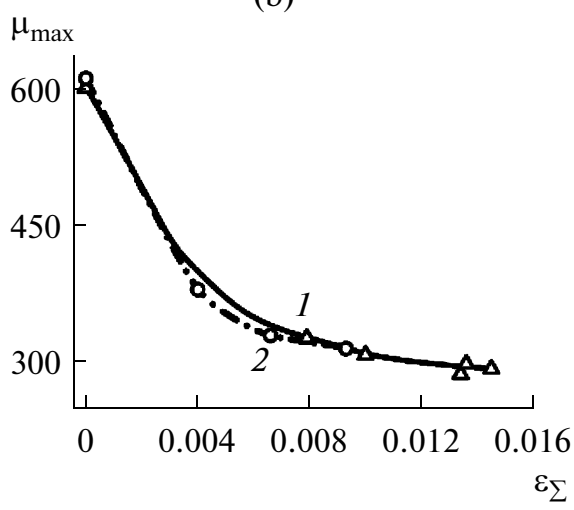

Fig. 8. The dependences of the maximum magnetic permeability on (a) the number of loading cycles and (b) the value of the accumulated plastic strain under cyclic loading of steel 50: (1) after heat treatment (quenching + tempering at $350^{\circ} \mathrm{C}$ ) and (2) after combined SHT of quenched steel (frictional treatment + tempering at $350^{\circ} \mathrm{C}$ ).
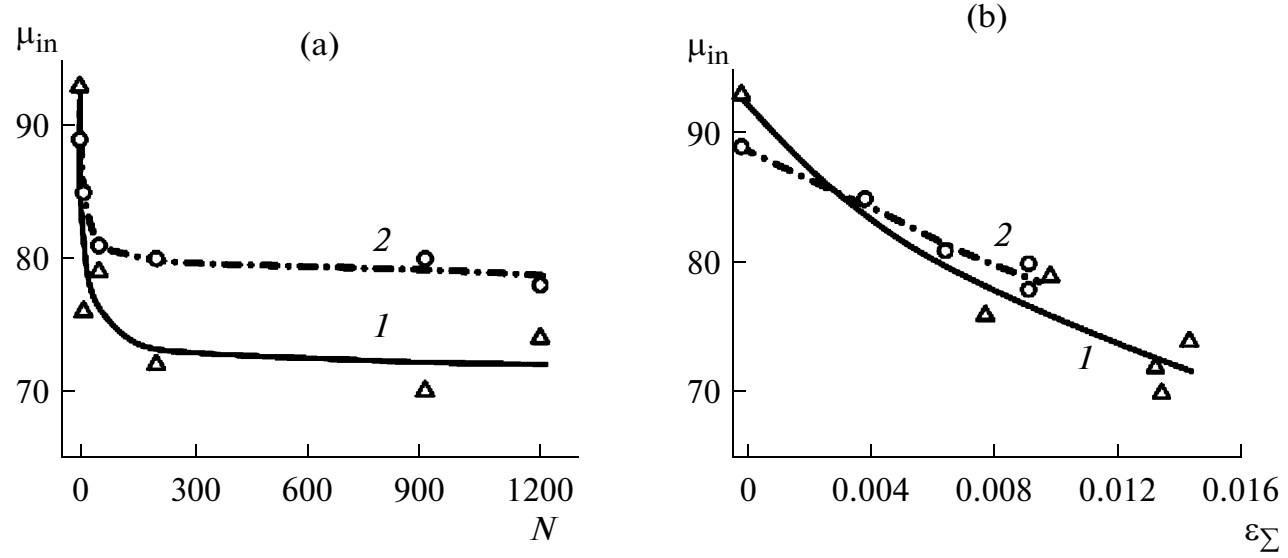

Fig. 9. The dependences of the initial magnetic permeability on (a) the number of loading cycles and (b) the value of the accumulated plastic strain under cyclic loading of steel 50: (1) after heat treatment (quenching + tempering at $350^{\circ} \mathrm{C}$ ) and (2) after combined SHT of quenched steel (frictional treatment + tempering at $350^{\circ} \mathrm{C}$ ).
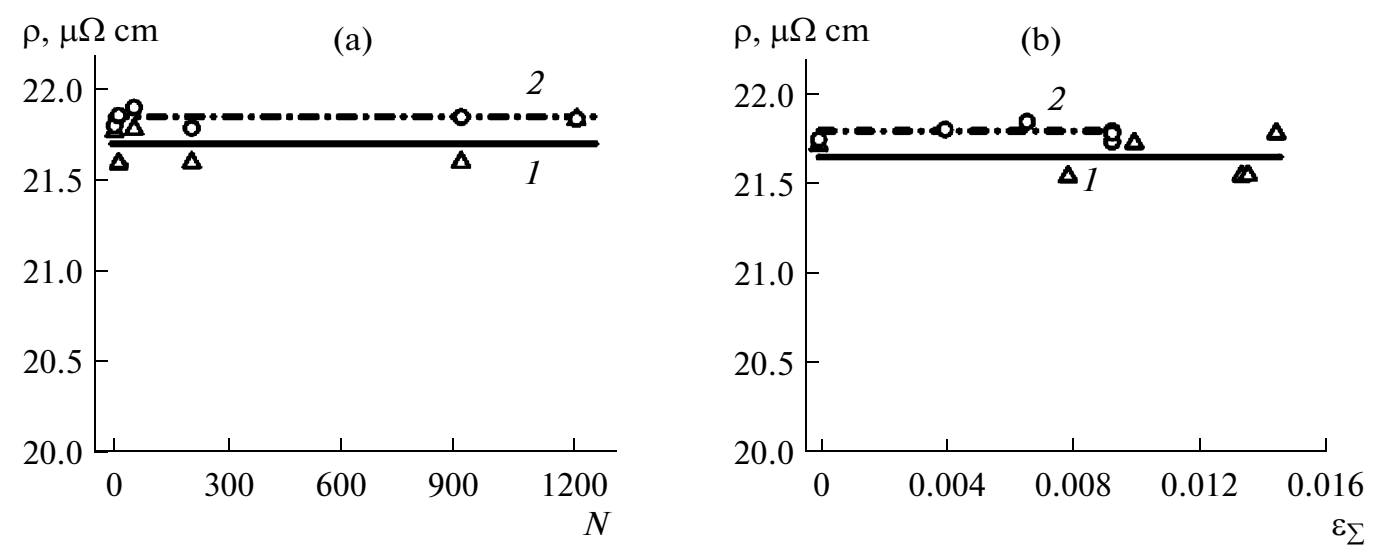

Fig.10. The dependences of the electrical resistivity on (a) the number of loading cycles and (b) the value of the accumulated plastic strain under cyclic loading of steel 50: (1) after heat treatment (quenching + tempering at $\left.350^{\circ} \mathrm{C}\right)$ and $(2)$ after combined SHT of quenched steel (frictional treatment + tempering at $350^{\circ} \mathrm{C}$ ). 
readings, $\alpha$, of the EC instrument sharply increased and as the loading continued, a substantial deceleration of the increase in the $\alpha$ value and its stabilization were observed.

The readings of EC transducers are proportional to the generalized EC parameter $\beta_{\mathrm{M}}$, which, for identical measurement conditions and weak exciting fields that correspond to the Rayleigh region, is determined only by the values of the initial permeability $\mu_{\text {in }}$ and electrical resistivity $\rho$ $\left(\alpha \sim \beta_{\mathrm{M}} \sim \sqrt{1 /\left(\mu_{\text {in }} \cdot \rho\right)}\right) \sim \beta_{\mathrm{M}} \sim \sqrt{1 /\left(\mu_{\text {in }} \cdot \rho\right)}$ [30].

Therefore, the aforementioned increase in the EC characteristics is evidently determined by a decrease in the initial permeability of heat-treated steel under "hard" cyclic loading (Fig. 9, curves 1), because the electrical resistivity remains virtually constant (Fig. 10, curves 1). The observed deceleration of the increase in the $\alpha$ value and its stabilization are also related to the value of the initial permeability $\mu_{\text {in }}$ and are determined by the fact that the value of $\mu_{\text {in }}$ changes slightly, when the number of loading cycles is $N>$ $50\left(\varepsilon_{\Sigma}>0.01\right)$ (Fig. 9, curves 1$)$.

In contrast, after the combined SHT, the character of the dependences of readings $\alpha$ of the EC instrument substantially differs for different excitation frequencies of the EC transducer (Fig. 11, curves 2). At a frequency $f=2.4 \mathrm{kHz}$ (Fig. 11, curves $\alpha_{1}$ ), an increase in the readings of the EC instrument is observed until the number of loading cycles reaches $N=200$ (the value of the accumulated plastic strain is $\varepsilon_{\Sigma}=$ 0.0093 ), and as the cyclic loading continues, the $\alpha$ value drops slightly. At frequencies $f=24-108 \mathrm{kHz}$ (Fig. 11, curves $\alpha_{3}-\alpha_{7}$ ), a minimum is observed in the analyzed dependences 2 for the number of cycles $N=10\left(\varepsilon_{\Sigma}=0.0040\right)$. This minimum becomes more pronounced as the excitation frequency of the EC transducer increases. As the loading continues to a number of cycles of $N=200$, the $\alpha$ value increases; at frequencies $f$ of 72 (curves $\alpha_{5}$ ), 96 (curves $\alpha_{6}$ ), and $108 \mathrm{kHz}$ (curves $\alpha_{7}$ ), an intense increase in $\alpha$ is observed only to a number of cycles of $N=50\left(\varepsilon_{\Sigma}=0.0066\right)$. For $N>200$, the $\alpha$ value decreases at frequencies of $f=12-108 \mathrm{kHz}$, as well as at $2.4 \mathrm{kHz}$ (Fig. 11a, curves 2).

The observed change in the character of dependences 2 of readings $\alpha$ of the EC instrument is determined by different values of the electromagnetic-field penetration depth $\delta$, which is proportional to $\sqrt{1 / f}$ (the larger $f$ is, the smaller $\delta$ is) [31]. At the minimum frequency $f=2.4 \mathrm{kHz}$, the thickness of the investigated layer $(\delta \sim 800 \mu \mathrm{m})$ appreciably exceeds the thickness of the hardened layer $(h \sim 220 \mu \mathrm{m})$ and the readings of the EC instrument are determined mainly by the properties of the nonhardened base (Fig. 11, curves $\alpha_{1}$ ). However, as the excitation frequency of the EC transducer increases, the contribution of the properties of the thin hardened layer to the $\alpha$ value becomes more substantial (Fig. 11, curves $\alpha_{2}-\alpha_{7}$ ). At a frequency of $f=24 \mathrm{kHz}$, the depth of the analyzed layer $(\delta \sim 220 \mu \mathrm{m})$ corresponds to the hardened-layer thickness, whereas at the maximum operating frequency $(f=108 \mathrm{kHz})$, the analyzed layer $(\delta \sim 120 \mu \mathrm{m})$ is equal to approximately half of the hardened surface layer.

The minimum for a number of cycles $N=10$ and a decrease in $\alpha$ for $N>200$ in the considered dependences (Fig. 11a, curves 2) may be associated with cracking during cyclic loading of the surface layer, which was hardened using combined SHT (Fig. 5a, 5b). Continuity violations increase the resistance of the metal, thus reducing ECs. It was theoretically shown in [32] that a change in the resistivity of the conducting material due to cracks is very significant even for a small number of cracks (Fig. 12). Therefore, the pronounced minimum in the readings $\alpha$ of the EC transducer for a number of cycles of $N=10$ (Fig. 11, curves 2), which is observed despite a decrease in the initial permeability (Fig. 9, curves 2), results from an abrupt increase in the resistivity of the thin steel surface layer at the initial cracking stage (Fig. 5a). In this case, the contribution of the electrical resistivity to the $\alpha$ value prevails over the influence of the initial permeability. It should be noted that the increase in the resistivity of the thin surface layer, which is caused by cracking, does not manifest itself in bulk measurements of the resistivity of specimens after the SHT and subsequent cyclic loading (Fig. 10, curves 2), when the contribution of the nonhardened steel base predominates.

Thus, in order to test the initial stages of cracking under cyclic loading of quenched steel 50 that was strengthened using the SHT, it is desirable to use high excitation frequencies of the EC transducer $(f=72-$ $108 \mathrm{kHz}$ ), when the analyzed-layer depth is smaller than the hardened-layer thickness. In contrast, the EC testing of the accumulated plastic strain should be performed at the minimum frequency $(f=2.4 \mathrm{kHz})$, when the contribution of cracking in the hardened layer to the EC characteristics is insignificant and a close correlation between changes in the readings of the EC instrument (Fig. 11a, $\alpha_{1}$, curve 2 ) and the accumulated plastic strain (Fig. 3b, curve 2) is observed upon an increase in the number of loading cycles to $N=200$ (to a value of the accumulated plastic strain of $\varepsilon_{\Sigma}=0.0093$ ).

The increase in the readings $\alpha$ of the EC instrument for the steel 50 that was subjected to combined SHT with an increase in the number of loading cycles from $N=10$ to $N=200$ (Fig. 11a, curves 2) is due 
(a)

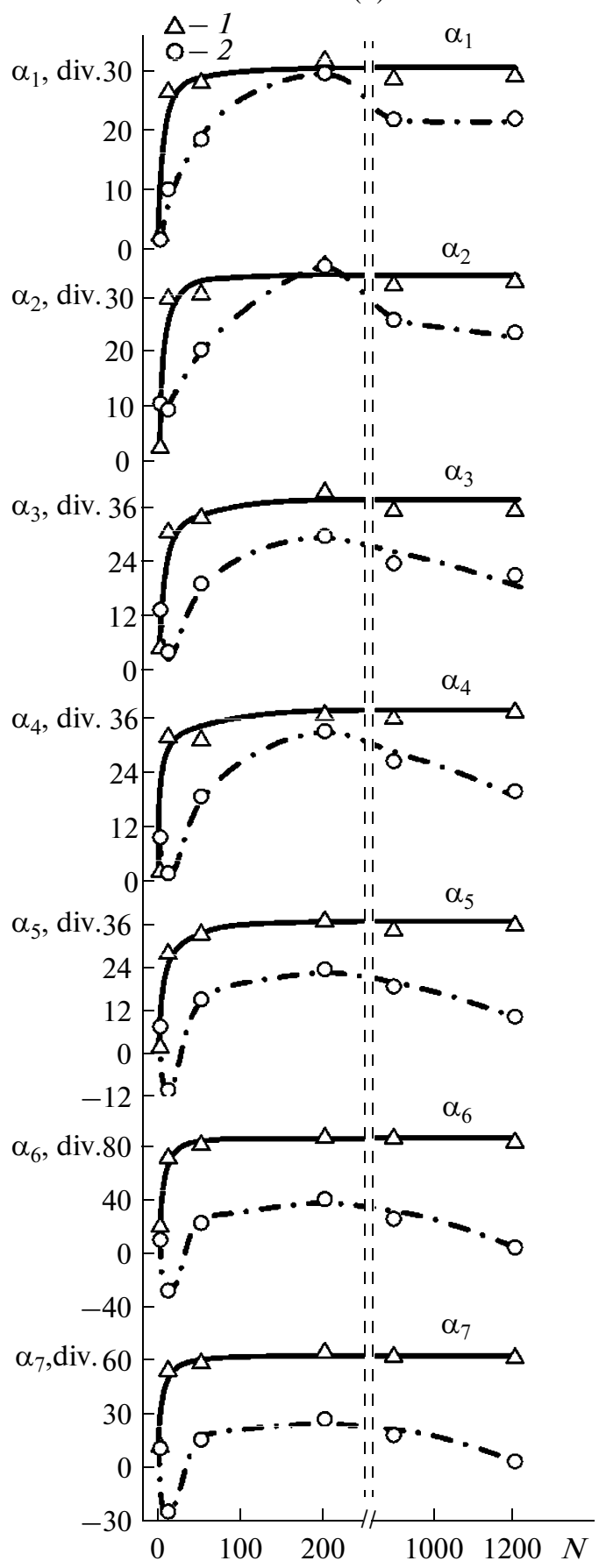

(b)

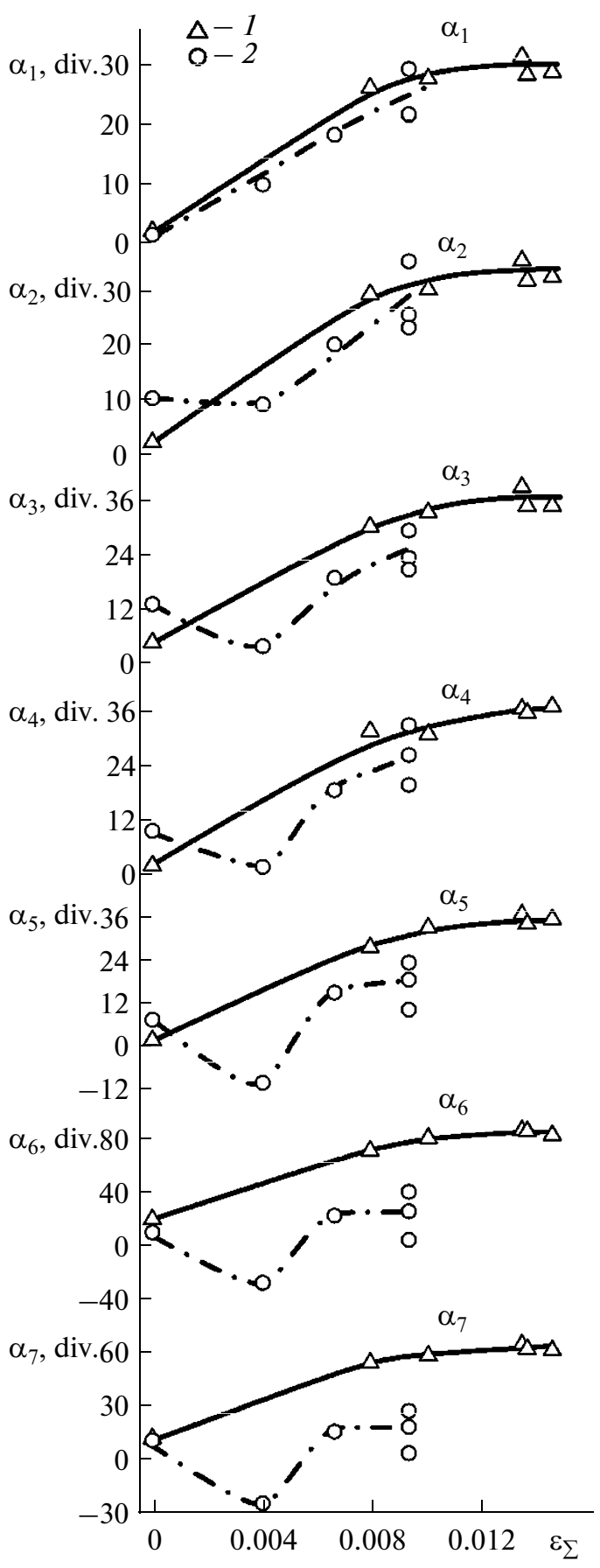

Fig. 11. The dependences of readings of the EC instrument at frequencies of $2.4\left(\alpha_{1}\right), 12\left(\alpha_{2}\right), 24\left(\alpha_{3}\right), 36\left(\alpha_{4}\right), 72\left(\alpha_{5}\right)$, $96\left(\alpha_{6}\right)$, and $108 \mathrm{kHz}\left(\alpha_{7}\right)$ on (a) the number of loading cycles and (b) the value of the accumulated plastic strain under cyclic loading of steel 50: (I) after heat treatment (quenching + tempering at $350^{\circ} \mathrm{C}$ ) and (2) after combined SHT of quenched steel (frictional treatment + tempering at $350^{\circ} \mathrm{C}$ ).

to the predominating influence of the initial permeability, which decreases by a larger value (Fig. 9, curves 2 ) upon accumulation of larger (than for $N=10$ ) plastic strain (Fig. 3b, curve 2). The decrease in the $\alpha$ value for the number of cycles $N>200$ in the considered curves is related to the ongoing cracking of the surface layer (Fig. 5b) and the corresponding increase in the resistivity, while no additional strain accumulation 


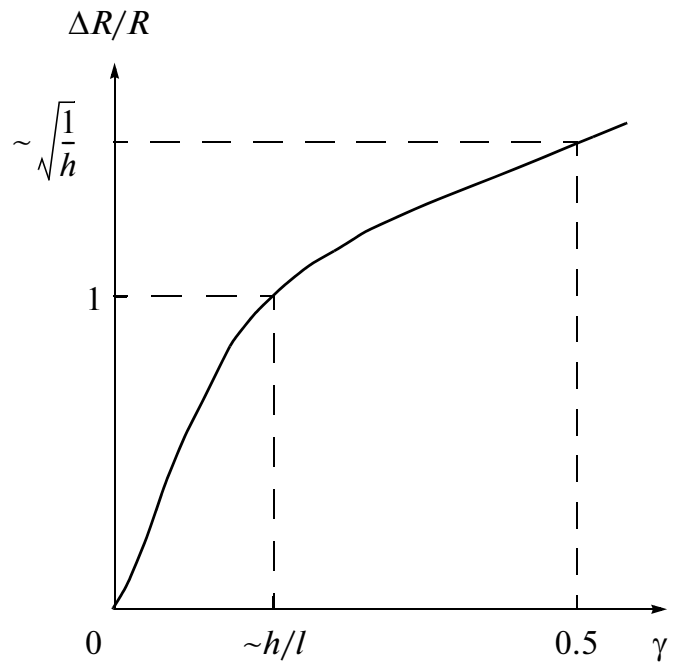

Fig. 12. The change in the resistance, $\Delta R / R$, as a function of cracking $\gamma=2 l h / r_{c}^{2}$ of the conducting material ( $l$ is the crack length, $h$ is the crack opening, and $r_{c}$ is the average distance between the centers of cracks) [32].

(Fig. 3b, curve 2) and the corresponding decrease in the initial permeability occur. In fact, Fig. 9 shows that, as in the case of heat-treated specimens (curves 1 ), the value of $\mu_{\text {in }}$ changes slightly for specimens after SHT (curves 2) for the number of cycles $N>200$.

The qualitatively identical character of the dependences of readings $\alpha$ on the number of loading cycles (the value of the accumulated plastic strain) for steel 50 specimens after heat treatment (Fig. 11, curves 1), which is observed for all excitation frequencies of the EC transducer, is determined by the fact that cracking, which is characteristic of the specimens that were subjected to the SHT, does not occur (Fig. 5c, 5d).

\section{CONCLUSIONS}

The possibilities of using the magnetic and EC methods to test the fatigue degradation of quenched steel $50(0.51 \% \mathrm{C})$, which was initially subjected to the combined nanostructuring SHT (including frictional treatment with the subsequent tempering at $T=350^{\circ} \mathrm{C}$ and providing a good combination of the wear resistance, strength, and plasticity), under low-cycle fatigue loading were established.

It was shown that the plastic strain that was accumulated under cyclic loading can be tested by measuring (i) the coercive force and residual induction on the major and minor magnetic-hysteresis loops at maximum magnetic inductions in hysteresis cycles of 1 and $0.4 \mathrm{~T}$ to the value of the accumulated plastic strain $\varepsilon_{\Sigma}=0.0093$ and at maximum magnetic inductions in hysteresis cycles of 0.1 and $0.05 \mathrm{~T}$ to $\varepsilon_{\Sigma}=0.004$; and (ii) by measuring the maximum and initial permeabilities to $\varepsilon_{\Sigma}=0.0066$.

It was found that under "hard" cyclic loading of quenched steel that was subjected to the SHT, cracks appear on the surfaces of specimens. The length and number of cracks increased as the number of loading cycles increased. It was shown that the EC technique can be used to test the initial stages of crack formation $\left(\varepsilon_{\Sigma} \leq 0.004\right)$ by using frequencies of $24-108 \mathrm{kHz}$, when the calculated depth of the analyzed layer $(220-120 \mu \mathrm{m})$ does not exceed the depth of the frictional-treatment-hardened layer $(\sim 220 \mu \mathrm{m})$. This is based on the discovered effect of a decrease in the readings of the EC instrument, which is associated with an increase in the resistivity of a thin surface layer during crack formation. In this case, on the initial segment of the dependences of readings of the EC instrument on the number of loading cycles (the value of the accumulated plastic strain), a minimum is observed, which becomes more pronounced with an increase in the excitation frequency of the EC transducer and a corresponding decrease in the depth of the analyzed layer.

It was shown that the plastic strain accumulated during cyclic loading can be tested via taking readings of the EC instrument at a minimum frequency of $2.4 \mathrm{kHz}$ and a calculated analyzed-layer depth of $800 \mu \mathrm{m}$, when the contribution of the crack formation in the frictional-treatment-hardened layer to the EC characteristic is insignificant. 
These results can be used for predicting the fatigue degradation of articles produced from medium-carbon steels under cyclic loading that were subjected to combined SHT.

\section{ACKNOWLEDGMENTS}

This study was supported in part by the Program of Basic Research of the Ural Branch, Russian Academy of Sciences, project no. 12-P-1-1027 (according to the Program of the Presidium of the Russian Academy of Sciences no. 25) and the Russian Foundation for Basic Research, projects nos. 11-08-01025-a and 13-01-00732_a.

Mechanical tests, electron scanning microscopy, profilometry, and magnetic measurements were performed at the CKP Plastometry of IES, Ural Branch, Russian Academy of Sciences. The electron transmission microscopy was performed on the equipment of the Laboratory of Structural Methods for Analysis of Materials and Nanomaterials, CKP, Ural Federal University.

\section{REFERENCES}

1. Makarov, A.V., Nanostructuring frictional treatment of carbon and low-alloyed steels, in Perspektivnye materialy. T. IV: Uchebnoe posobie (Promising Materials: Tutorial), Merson, D.L., Ed., Tolyatti: TGU, 2011, vol. IV, pp. 123-207.

2. Makarov, A.V., Pozdejeva, N.A., Savrai, R.A., Yurovskikh, A.S., and Malygina, I.Yu., Influence of frictional and combined strain-heat treatments on the tribological and mechanical properties of hardened constructional steel, Izv. Samar. Nauch. Tsentr. Ross. Akad. Nauk., 2011, vol. 13, no. 4(3), pp. 799-804.

3. Makarov, A.V., Gorkunov, E.S., Kogan, L.Kh., and Malygina, I.Yu., Estimation of the quality of strengthening frictional treatment and subsequent tempering of eutectoid steel by the eddy-current method, Russ. J. Nondestr. Test., 2009, vol. 45, no. 2, pp. 133-142.

4. Makarov, A.V., Gorkunov, E.S., Savrai, R.A., Kolobylin, Yu.M., Kogan, L.Kh., Yurovskikh, A.S., Pozdejeva, N.A., and Malygina, I.Yu., The peculiarities of magnetic and eddy-current testing of quenched structural steel hardened by nanostructuring frictional treatment, Russ. J. Nondestr. Test., 2012, vol. 48, no. 11, pp. 615-622.

5. Makarov, A.V., Gorkunov, E.S., Savrai, R.A., Kolobylin, Yu.M., Kogan, L.Kh., Pozdejeva, N.A., and Malygina, I.Yu., Magnetic and eddy-current testing of hardened constructional steel subjected to combined strain-thermal treatment, Russ. J. Nondestr. Test., 2012, vol. 48, no. 12, pp. 673-685.

6. Donzella, G. and Granzotto, S., Some experimental results about the correlation between Barkhausen noise and the fatigue life of steel specimens, J. Magnet. Magnet. Mater., 1994, vol. 133, pp. 613-616.

7. Palma, E.S., Mansur, T.R., Ferreira, SilvaS.Jr., and Alvarenga, A., Fatigue damage assessment in AISI 8620 steel using Barkhausen noise, Int. J. Fatig., 2005, vol. 27, pp 659-665.

8. Gorkunov, E.S., Savrai, R.A., Makarov, A.V., Zadvorkin, S.M., Smirnov, S.V., Rogovaya, S.A., and Solomein, M.N., Application of magnetic and electromagnetic-acoustic methods for assessing plastic deformations under cyclic loading of annealed intermediate-carbon steel, Russ. J. Nondestr. Test., 2006, vol. 42, no. 5, pp. 309-314.

9. Gorkunov, E.S., Savrai, R.A., Makarov, A.V., Kogan, L.Kh., and Rogovaya, S.A., Application of an eddy-current method for the assessment of stored plastic deformation and residual mechanical properties after cyclic loading of an annealed medium-carbon steel, Russ. J. Nondestr. Test., 2007, vol. 43, no. 4, pp. 228-233.

10. Bystrushkin, G.S., Study of the possibility of determining the early stage of fatigue damage of chromium steel using the eddy-current method, Defektoskopiya, 1968, no. 5, pp. 1-7.

11. Makarov, A.V., Savrai, R.A., Gorkunov, E.S., Malygina, I.Yu., Kogan, L.Kh., Pozdejeva N.A., and Kolobylin, Yu.M., Effect of friction-induced hardening on the features of magnetic and eddy-current behavior of an annealed structural steel under cyclic loading conditions, Russ. J. Nondestr. Test., 2008, vol. 44, no. 7, pp. 496-508.

12. Shcherbinin, V.E. and Gorkunov, E.S., Magnitnyi kontrol' kachestva metallov (Magnetic Quality Control of Metals), Yekaterinburg: UrO RA N, 1996.

13. Makarov, A.V., Gorkunov, E.S., Kogan, L.Kh., Kolobylin, Yu.M., Korshunov, L.G., and Osintseva, A.L., Features of electromagnetic methods for testing the wear resistance of medium-carbon structural steel subjected to laser or bulk hardening and tempering, Russ. J. Nondestr. Test., 2006, vol. 42, no. 7, pp. 443-451.

14. Kragel'skii, I.V., Dobychin, M.N., and Kombalov, V.S., Osnovy raschetov na trenie i iznos (Fundamentals of Friction and Wear Calculations), Moscow: Mashinostroenie, 1977.

15. Makarov, A.V., Savrai, R.A., Pozdejeva, N.A., Smirnov, S.V., Vichuzhanin, D.I., Korshunov, L.G., and Malygina, I.Yu., Effect of hardening friction treatment with hard-alloy indenter on microstructure, mechanical properties, and deformation and fracture features of constructional steel under static and cyclic tension, Surf. Coat. Technol., 2010, vol. 205, no. 3, pp. 841-852. 
16. Vychuzhanin D.I., Makarov A.V., Smirnov S.V., Pozdeeva N.A., and Malygina I.Yu., Stress and strain and damage during frictional strengthening treatment of flat steel surface with a sliding cylindrical indenter, J. Machin. Manufact. Reliabil., 2011, vol. 40, no. 6, pp. 554-560.

17. Makarov, A.V., Pozdejeva, N.A., Savrai R.A., Yurovskikh A.S., and Malygina I.Yu., Improvement of wear resistance of quenched structural steel by nanostructuring frictional treatment, J. Frict. Wear, 2012, vol. 33, no. 6, pp. 433-442.

18. Klesnil, M. and Lukáš, P., Fatigue of Metallic Materials. Second Edition. Materials Science Monographs, Elsevier, 1992, vol. 71.

19. Terent'ev, V.F., Ustalostnaya prochnost' metallov i splavov (Fatigue Strength of Metals and Alloys), Moscow: Intermet Inzhiniring, 2002.

20. Panin, V.E., Slosman, A.I., Antipina, N.A., and Litvinenko, A.V., Effect of the internal structure and surface state on the strain development at the mesolevel of low-carbon steel, Fizich. Mezomekh., 2001, vol. 4, no. 1, pp. 105-110.

21. Klimenov, V.A., Panin, S.V., and Bezborodov, V.P., Studying the strain character at the mesoscale level and destruction of a gas-thermal coating-base composition under tension, Fizich. Mezomekh., 1999, vol. 2, no. 12, pp. 141-156.

22. Panin, V.E., Elsukova, T.F., and Popkova, Yu.F., Staging of multilevel development of fatigue cracks as a nonlinear rotational-type self-sustained wave process, Fizich. Mezomekh., 2010, vol. 13, no. 6, pp. 13-25.

23. Mikheev, M.N. and Gorkunov, E.S., Magnitnye metody strukturnogo analiza i nerazrushayushchego kontrolya (Magnetic Methods for Structural Analysis and Nondestructive Testing), Moscow: Nauka, 1993.

24. Jiles, D.C., The effect of compressive plastic deformation of AISI 4130 steels with various microstructures, J. Phys. D, Appl. Phys., 1988, no. 21, pp. 1196-1204.

25. Zaikova, V.A., Startseva, I.E., and Filippov, B.N., Domennaya struktura i magnitnye svoistva elektrotekhnicheskikh stalei (Domain Structure and Magnetic Properties of Electrical Steels), Moscow: Nauka, 1992.

26. Hetherington, M.G., Jakubovics, J.P., Szpunar, J.A., and Tanner, B.K., High-voltage Lorentz electron microscopy studies of domain structures and magnetization processes in pearlitic steels, Philosoph. Magaz., B, 1987, vol. 56, no. 5 , pp. 561-577.

27. Troible, G. and Zeger, A., Influence of crystal-lattice defects on magnetization processes in ferromagnetic single crystals, in Plasticheskaya deformatsiya monokristallov (Plastic Strain of Single Crystals), Moscow: Mir, 1996, pp. 201-264.

28. Kuleev, V.G., Tsar'kova, T.P., and Nichipuruk, A.P., Specific features of the behavior of the coercive force in low-carbon plastically deformed steels, Russ. J. Nondestr. Test., 2005, vol. 41, no. 5, pp. 285-295.

29. Kuleev, V.G., Tsar'kova, T.P., and Nichipuruk, A.P., Effect of tensile plastic strains on the residual magnetization and initial permeability of low-carbon steels, Russ. J. Nondestr. Test., 2006, vol. 42, no. 4, pp. $261-271$.

30. Dyakin, V.V. and Sandovskii, V.A., Teoriya i raschet nakladnykh vikhretokovykh preobrazovatelei (Theory and Calculation of Attachable Eddy-Current Transducers), Moscow: Nauka, 1981.

31. Dorofeev A.L., Induktsionnaya strukturoskopiya (Induction Structuroscopy), Moscow: Energiya, 1973.

32. Fel'dman, E.P., Vinnik, E.A., Granovskii, Ya.I., and Kalugina, N.A., Elektrosoprotivlenie Provodyashchego materiala, soderzhashchego treshchiny. Fiz.-Tekhn. Probl. Gorn. Proizvod., 2011, no. 14, pp. 54-59.

Translated by A. Seferov 